\title{
Comparison of Glycemic Control Indicators and Safety Evaluation during Linagliptin Treatment over 6 Months in Japanese Type 2 Diabetic Patients with and without Nephropathy
}

Masami Tanaka*, Takeshi Nishimura, Risa Sekioka, Toshihide Kawai, Shu Meguro, Junichiro Irie, Yoshifumi Saisho and Hiroshi Itoh

Department of Internal Medicine, School of Medicine, Keio University, 35 Shinanomachi, Shinjuku-ku, Tokyo 160-8582, Japan

\begin{abstract}
Objective: This retrospective study was undertaken to compare hemoglobin A1c and glycoalbumin levels as glycemic control indicators during linagliptin treatment in diabetic patients with or without nephropathy. The efficacy and safety of linagliptin were also examined.

Methods: The subjects were 127 outpatients with type 2 diabetes, including 69 patients with nephropathy. The hypoglycemic effect of linagliptin and the factors contributing to its hypoglycemic effect were examined. Several clinical parameters were compared before and after the initiation of linagliptin to evaluate the drug's safety.

Results: Linagliptin significantly decreased hemoglobin A1c and glycoalbumin levels at 3 and 6 months after treatment initiation. At 6 months, changes in hemoglobin A1c levels from baseline were strongly correlated with changes in glycoalbumin levels in diabetic patients with and without nephropathy. Changes in hemoglobin A1c and glycoalbumin at 6 months were significantly greater in patients with higher baseline values and shorter diabetes duration. Linagliptin decreased both hemoglobin A1c and glycoalbumin levels, irrespective of the baseline estimated glomerular filtration rate. No changes in clinical parameters thought to indicate adverse events were noted.
\end{abstract}

Conclusions: Glycoalbumin is an equivalent glycemic control indicator and predictor to hemoglobin A1c during linagliptin treatment. Linagliptin is safe and effective in diabetic patients with or without nephropathy.

Keywords: Linagliptin; Hemoglobin A1c; Glycoalbumin; Type 2 diabetes mellitus; Diabetic nephropathy

\section{Introduction}

Dipeptidyl peptidase-4 (DPP-4) inhibitors are widely used to treat diabetes because of their robust hypoglycemic effect and the minimal risks of hypoglycemia and weight gain [1]. As such, they occupy an important position in any diabetes treatment strategy.

Hemoglobin Alc (HbAlc) has been the gold standard for evaluating glycemic control in patients with diabetes mellitus since the Diabetes Control and Complications Trial and United Kingdom Prospective Diabetes Study established its importance as an outcome predictor. However, some diabetes patients develop vascular complications despite relatively low HbAlc levels. Recent studies have suggested that vascular complications are caused by increased glycemic variability [2]. Although postprandial hyperglycemia, which is the cause of glycemic variability, is frequently observed in patients with uncontrolled diabetes, it has also been reported in diabetes patients with intermediate HbAlc levels [3,4].

As glycoalbumin (GA) is generated 4-6 times faster than $\mathrm{HbAlc}$, it responds to changes in blood glucose levels faster than HbAlc. It is suggested that GA could be a better marker for glycemic excursion, which is considered to be a major cause of atherosclerosis, than $\mathrm{HbAlc}$ [5]. A study using continuous glucose monitoring revealed that GA but not $\mathrm{HbAlc}$ is associated with blood glucose variability [6]. In addition, HbAlc appears to be less precise in patients with advanced stages of chronic kidney disease, particularly in patients with anemia treated with erythropoietin [7]. This phenomenon might reflect shortened red blood cell survival in patients with advanced kidney disease. In these patients, the time for glucose and hemoglobin to chemically interact is shortened [8].
In contrast to other DPP-4 inhibitors, linagliptin is excreted unchanged in bile and stool, which makes dose adjustment unnecessary in patients with renal dysfunction $[9,10]$. As conventional oral hypoglycemic agents have limitations regarding their administration to patients with advanced kidney disease, the prescription of linagliptin is expected to increase in these patients. Moreover, as $\mathrm{GA}$ is more precise than $\mathrm{HbAlc}$ in patients with severe renal impairment, GA will likely be measured more frequently than $\mathrm{HbA1c}$ in diabetes patients receiving linagliptin. Consequently, comparing the usefulness of GA with that of $\mathrm{HbA} 1 \mathrm{c}$ as indicators of glycemic control in diabetes patients with renal dysfunction is clinically relevant.

Therefore, we retrospectively evaluated the usefulness of $\mathrm{HbAlc}$ and GA as glycemic control indicators and predictors during linagliptin treatment in diabetes patients with and without diabetic nephropathy. In addition, we investigated the efficacy and safety of linagliptin, in particular in patients with a low estimated glomerular filtration rate (eGFR).

*Corresponding author: Dr. Masami Tanaka, Department of Internal Medicine School of Medicine, Keio University, 35 Shinanomachi, Shinjuku-ku, Tokyo 1608582, Japan, Tel: 81-3-5363-3797; Fax: 8133359-2745; E-mail: tana176k@keio.jp

Received February 01, 2016; Accepted February 16, 2016; Published February 23, 2016

Citation: Tanaka M, Nishimura T, Sekioka R, Kawai T, Meguro S, et al. (2016 Comparison of Glycemic Control Indicators and Safety Evaluation during Linagliptin Treatment over 6 Months in Japanese Type 2 Diabetic Patients with and without Nephropathy. J Diabetes Metab 7: 648. doi:10.4172/2155-6156.1000648

Copyright: $\odot 2016$ Tanaka M, et al. This is an open-access article distributed unde the terms of the Creative Commons Attribution License, which permits unrestricted use, distribution, and reproduction in any medium, provided the original author and source are credited. 
Citation: Tanaka M, Nishimura T, Sekioka R, Kawai T, Meguro S, et al. (2016) Comparison of Glycemic Control Indicators and Safety Evaluation during Linagliptin Treatment over 6 Months in Japanese Type 2 Diabetic Patients with and without Nephropathy. J Diabetes Metab 7: 648. doi:10.4172/2155-6156.1000648

Page 2 of 6

\section{Materials and Methods}

\section{Patients}

The subjects were 127 outpatients with type 2 diabetes who started linagliptin treatment from March 1, 2012 to March 31, 2014 at our hospital and continued the treatment for more than 6 months.

\section{Compliance with ethical standards}

This study was approved by the Ethics Committee of the Keio University School of Medicine and was performed in accordance with the Declaration of Helsinki.

\section{Data collection}

In this retrospective study, basic demographic data were collected from medical records, including sex, age, height, weight, diabetes duration, HbAlc, GA, blood glucose, C-peptide immunoreactivity (CPR), and hemoglobinlevels, eGFR, lipid profile (total cholesterol [TC], high-density lipoprotein [HDL] cholesterol, low-density lipoprotein [LDL] cholesterol, and triglycerides levels), and liver function enzymes (aspartate aminotransferase [AST], alanine aminotransferase [ALT], and $\gamma$-glutamyl transpeptidase $[\gamma$-GTP] levels). Number of sampling for GA is as same as that for HbAlc. Furthermore, the CPR index, which is thought to reflect $\beta$-cell function, was calculated as follows: plasma CPR $[\mathrm{ng} / \mathrm{mL}] /$ glucose $[\mathrm{mg} / \mathrm{dL}] \times 100$. All measurements were performed by the Department of Laboratory Medicine of the Keio University School of Medicine using routine automated laboratory methods [11]. Data on complications such as hypertension (blood pressure $\geq 140 / 90 \mathrm{mmHg}$ and/or the use of antihypertensive drugs) and dyslipidemia (TC $\geq 220 \mathrm{mg} / \mathrm{dL}$ and/or HDL cholesterol $<40 \mathrm{mg} /$ $\mathrm{dL}$ and/or triglycerides $\geq 150 \mathrm{mg} / \mathrm{dL}$ and/or taking a hypolipidemic agent) were also collected. Fasting was not required at blood sample collection. Body mass index (BMI) was calculated as weight divided by height squared $\left(\mathrm{kg} / \mathrm{m}^{2}\right)$.

Diabetic retinopathy was assessed through dilated pupils by an ophthalmologist using an ophthalmoscope. It was classified according to the modified Davis classification as no diabetic retinopathy, simple diabetic retinopathy, preproliferative diabetic retinopathy, or proliferative diabetic retinopathy [12]. Diabetic nephropathy was defined as urinary albumin excretion $\geq 30 \mathrm{mg} / \mathrm{g}$ creatinine or eGFR $<30 \mathrm{~mL} \cdot \mathrm{min}^{-1} \cdot 1.73 \mathrm{~m}^{-2}$. The degree of diabetic nephropathy was graded according to the new classification of diabetic nephropathy of the Japanese Diabetes Society [13]. Prior history of cerebrovascular disease, cardiovascular disease, and peripheral vascular disease was recorded. HbAlc levels are expressed in accordance with the National Glycohemoglobin Standardization Program guidelines, as recommended by the Japanese Diabetes Society [14]. The eGFR was calculated using the following formula established by the working group of the Japanese Chronic Kidney Disease Initiative: eGFR $\left(\mathrm{mL} \cdot \mathrm{min}^{-1} \cdot 1.73 \mathrm{~m}^{-2}\right)=194 \times($ serum creatinine $)-1.094 \times($ age $)-0.287$ $(\times 0.739$ for women) [15].

\section{Statistical analysis}

We retrospectively compared $\mathrm{HbAlc}$ and GA levels at baseline (at the start of linagliptin treatment), 3 months, and 6 months. Changes in $\mathrm{HbA} 1 \mathrm{c}$ and GA levels from baseline were analyzed by repeated-measures ANOVA; post hoc pairwise group comparisons were conducted using the Bonferroni test. Changes in hemoglobin levels were analyzed by the same method. $\triangle \mathrm{HbAlc}$ was defined as HbAlc levels at 6 months - baseline HbA1c levels; likewise, $\Delta \mathrm{GA}$ was defined as GA levels at 6 months - baseline GA levels. Correlations between $\Delta \mathrm{HbAlc}$ and $\Delta \mathrm{GA}$, $\triangle \mathrm{HbA} 1 \mathrm{c}$ and baseline eGFR, and $\triangle \mathrm{GA}$ and baseline eGFR were tested using Pearson's correlation coefficients. Linear regression analysis was used to identify independent predictors of $\Delta \mathrm{HbAlc}$ and $\triangle \mathrm{GA}$. The following considered clinically meaningful variables were used as independent variables in the multivariate analyses: baseline CPR index, baseline eGFR, sex, age, BMI, diabetes duration, and baseline HbAlc levels for $\triangle \mathrm{HbAlc}$ or baseline GA levels for $\Delta \mathrm{GA}$.

To evaluate the safety of linagliptin, several parameters were compared between baseline and 6 months after linagliptin administration. A paired $t$-test was used to compare normally distributed clinical parameters (body weight, hemoglobin, AST, ALT, TC, HDL cholesterol, LDL cholesterol, uric acid levels, and $\gamma$-GTP levels, CPR index, and eGFR). The Wilcoxon signed-rank test was used to compare non-normally distributed clinical parameters (i.e., triglycerides). These tests were performed for all patients $(n=127)$ and those with diabetic nephropathy $(n=69)$. Changes in the eGFR after linagliptin administration were analyzed using repeated-measures ANOVA in 3 patient categories stratified by baseline eGFR $(<30,30$ 60 , and $\geq 60 \mathrm{~mL} \cdot \mathrm{min}^{-1} \cdot 1.73 \mathrm{~m}^{-2}$ ).

All analyses were performed with SPSS version 19.0 (SPSS Inc, Chicago, IL, USA). Data are expressed as mean \pm SD, and the level of significance was set at $P<0.05$.

\section{Results}

\section{Baseline patient demographic characteristics}

The baseline demographic characteristics of the study subjects are shown in Table 1. The patients' mean eGFR was $54.2 \mathrm{~mL} \cdot \mathrm{min}^{-1} \cdot 1.73$ $\mathrm{m}^{-2}$, and 69 patients had diabetic nephropathy. Based on these findings, many of the study participants were likely to meet the chronic kidney disease criteria. Five patients were under erythropoietin therapy.

\section{Changes in hemoglobin A1c and glycoalbumin levels after linagliptin administration}

Changes in HbAlc and GA levels are shown in Figure 1. In all patients (Figure 1a), HbAlc and GA levels decreased significantly at 3 and 6 months after the start of linagliptin administration (baseline $\rightarrow$ 3 months $\rightarrow 6$ months: HbAlc $7.8 \pm 1.5 \% \rightarrow 7.3 \pm 1.3 \rightarrow 7.4 \pm 1.4$; GA $21.6 \pm 6.2 \rightarrow 19.9 \pm 4.6 \% \rightarrow 20.1 \pm 5.2 \%$; all $P<0.001$ vs. baseline). In the 58 patients without diabetic nephropathy (Figure 1b), HbAlc and GA levels also decreased significantly at 3 and 6 months (HbA1c 7.5 $\pm 1.0 \% \rightarrow 7.1 \pm 1.0 \%, P<0.001$ vs. baseline $\rightarrow 7.1 \pm 1.0 \%, P<0.001$ vs. baseline; GA $19.7 \pm 3.4 \% \rightarrow 18.7 \pm 3.4, P=0.0048$ vs. baseline $\rightarrow 19.0 \pm$ $3.4, P=0.043$ vs. baseline). In the 69 patients with diabetic nephropathy (Figure 1c), HbA1c and GA levels also decreased significantly at 3 and 6 months (HbAlc $8.2 \pm 1.8 \% \rightarrow 7.5 \pm 1.5 \rightarrow 7.6 \pm 1.6$; GA $23.1 \pm 7.5 \% \rightarrow$ $20.8 \pm 5.3 ; \rightarrow 21.2 \pm 6.2$; all $P<0.001$ vs. baseline).

$\triangle \mathrm{GA}$ and $\triangle \mathrm{HbA} 1 \mathrm{c}$ were strongly correlated in all patient groups (total patients: $r=0.832, P<0.001$; patients without nephropathy: $r=$ 0.843, $P<0.001$; diabetic nephropathy patients: $r=0.828, P<0.001$; Figure 2$)$. Neither $\Delta \mathrm{GA}(r=0.111, P=0.216)$ nor $\Delta \mathrm{HbA1c}(r=0.085, P$ $=0.343)$ was significantly correlated with baseline eGFR.

Among the diabetic nephropathy patients whose hemoglobin level is less than $13 \mathrm{~g} / \mathrm{dL}, \mathrm{HbAlc}$ and GA levels decreased significantly at 3 and 6 months after the start of linagliptin administration (HbAlc 7.8 $\pm 1.8 \% \rightarrow 6.8 \pm 1.1 \%, P<0.001$ vs. baseline $\rightarrow 7.1 \pm 1.2 \%, P=0.0069$ vs. baseline; GA $23.8 \pm 8.3 \% \rightarrow 20.2 \pm 4.5 \%, P<0.001$ vs. baseline $\rightarrow$ $21.2 \pm 5.9 \%, P=0.013$ vs. baseline). Otherwise, among the diabetic nephropathy patients whose hemoglobin level is $13 \mathrm{~g} / \mathrm{dL}$ or above, 
Citation: Tanaka M, Nishimura T, Sekioka R, Kawai T, Meguro S, et al. (2016) Comparison of Glycemic Control Indicators and Safety Evaluation during Linagliptin Treatment over 6 Months in Japanese Type 2 Diabetic Patients with and without Nephropathy. J Diabetes Metab 7: 648. doi:10.4172/2155-6156.1000648

HbA1c level did not decrease significantly at 6 months and GA levels did not decrease significantly at both 3 and 6 months (HbAlc $8.7 \pm$ $1.8 \% \rightarrow 8.2 \pm 1.7 \%, P=0.015$ vs. baseline $\rightarrow 8.3 \pm 1.8 \%, P=0.055$ vs. baseline; GA $23.0 \pm 7.3 \% \rightarrow 21.5 \pm 5.7 \%, P=0.080$ vs. baseline $\rightarrow 21.8 \pm$ $6.6 \%, P=0.19$ vs. baseline).

In both patients with and without nephropathy, hemoglobin levels did not change significantly from baseline during the follow-up period (with nephropathy: $12.7 \pm 2.2 \mathrm{~g} / \mathrm{dL} \rightarrow 12.6 \pm 2.1 \mathrm{~g} / \mathrm{dL} \rightarrow 12.7 \pm 2.3 \mathrm{~g} / \mathrm{dL}$,

\begin{tabular}{|c|c|c|c|}
\hline \multicolumn{4}{|c|}{ Characteristic } \\
\hline$N$ & 127 & $\begin{array}{l}\text { Concomitant diabetic } \\
\text { therapy, } n(\%)\end{array}$ & $96(75.6)$ \\
\hline Male sex, $n(\%)$ & $79(62.2)$ & SU, $n(\%)$ & $22(17.3)$ \\
\hline Age (years) & $67.2 \pm 11.9$ & BG, $n(\%)$ & $20(15.7)$ \\
\hline $\begin{array}{c}\text { Diabetes duration } \\
\text { (years) }\end{array}$ & $15.0 \pm 9.8$ & TZD, $n(\%)$ & $3(2.4)$ \\
\hline BMI $\left(\mathrm{kg} / \mathrm{m}^{2}\right)$ & $25.2 \pm 4.7$ & $\alpha G I, n(\%)$ & $36(28.3)$ \\
\hline CPR index & $1.4 \pm 1.6$ & Glinide, $n(\%)$ & $14(11)$ \\
\hline $\mathrm{HbA} 1 \mathrm{c},(\%)$ & $7.9 \pm 1.5$ & Insulin, $n(\%)$ & $61(48)$ \\
\hline GA, $(\%)$ & $21.6 \pm 6.2$ & & \\
\hline AST, (U/L) & $24.1 \pm 15.5$ & Hypolipidemic agents, $n(\%)$ & $63(49.6)$ \\
\hline $\mathrm{ALT},(\mathrm{U} / \mathrm{L})$ & $21.2 \pm 18.1$ & Statin, $n(\%)$ & $59(46.5)$ \\
\hline eGFR, $\left(\mathrm{mL} / \mathrm{min} / 1.73 \mathrm{~m}^{2}\right)$ & $54.2 \pm 23.2$ & Fibrate, $n(\%)$ & $2(1.6)$ \\
\hline Retinopathy, $n(\%)$ & $49(39.8)$ & Ethyl icosapentate, $n(\%)$ & $4(3.1)$ \\
\hline NDR, $n(\%)$ & $74(60.2)$ & Ezetimibe, $n(\%)$ & $1(0.8)$ \\
\hline SDR, $n(\%)$ & $20(16.3)$ & Others, $n(\%)$ & $2(1.6)$ \\
\hline PPDR, $n(\%)$ & $7(3.6)$ & & \\
\hline PDR, $n(\%)$ & $22(17.9)$ & Antihypertensive drugs, $n(\%)$ & $84(66.1)$ \\
\hline Nephropathy, $n(\%)$ & $69(54.3)$ & $\mathrm{CCB}, n(\%)$ & $51(40.2)$ \\
\hline Stage $1, n(\%)$ & $58(45.7)$ & ARB, $n(\%)$ & $68(53.5)$ \\
\hline Stage $2, n(\%)$ & $28(22.0)$ & ACEI, $n(\%)$ & $4(3.1)$ \\
\hline Stage $3, n(\%)$ & $27(21.3)$ & Diuretics, $n(\%)$ & $26(20.5)$ \\
\hline Stage $4, n(\%)$ & $9(7.1)$ & a-blockers, $n(\%)$ & $2(1.6)$ \\
\hline Stage $5, n(\%)$ & $5(3.9)$ & $\beta$-blockers, $n(\%)$ & $22(17.3)$ \\
\hline Hypertension, $n(\%)$ & $88(69.3)$ & & \\
\hline Dyslipidemia, $n(\%)$ & $80(63.0)$ & $\begin{array}{l}\text { Antihyperuricemia drugs, } \\
n(\%)\end{array}$ & $24(18.9)$ \\
\hline Macroangiopathy, $n(\%)$ & $42(33.1)$ & Allopurinol, $n(\%)$ & $15(11.8)$ \\
\hline $\mathrm{CAD}, n(\%)$ & $20(15.7)$ & Febuxostat, $n(\%)$ & $7(5.5)$ \\
\hline CVD, $n(\%)$ & $27(21.3)$ & Others, $n(\%)$ & $2(1.6)$ \\
\hline ASO, $n(\%)$ & $2(1.6)$ & & \\
\hline & & Anticoagulant agents, $n(\%)$ & $10(7.9)$ \\
\hline & & Warfarin, $n(\%)$ & $9(7.1)$ \\
\hline & & Dabigatran, $n(\%)$ & $1(0.8)$ \\
\hline & & Antiplatelet agents, $n(\%)$ & $38(29.9)$ \\
\hline & & Aspirin, $n(\%)$ & $28(22)$ \\
\hline & & Clopidogrel, $n(\%)$ & $10(7.9)$ \\
\hline & & Ticlopidine, $n(\%)$ & $2(1.6)$ \\
\hline & & Cilostazol, $n(\%)$ & $6(4.7)$ \\
\hline
\end{tabular}

Values are expressed as number (\%) or mean \pm SD.

ACEI, angiotensin converting enzyme inhibitor; AST, aspartate aminotransferase; ALT, alanine aminotransferase; ARB, angiotensin receptor blocker; ASO arteriosclerosis obliterans; BG, biguanide; BMI, body mass index; CAD, coronary artery disease; CCB, calcium channel blocker; CPR, C peptide immunoreactivity CPR index $=100 \times$ serum CPR $(\mathrm{ng} / \mathrm{mL}) /$ blood glucose $(\mathrm{mg} / \mathrm{dL}) ;$ CVD, cerebral vascular disease; GA, glycoalbumin; $\alpha \mathrm{GI}$, a-glucosidase inhibitor; HbA1c, hemoglobin A1c; NDR, no diabetic retinopathy; PPDR, preproliferative diabetic retinopathy; PDR, proliferative diabetic retinopathy; SDR, simple diabetic retinopathy; SU, sulfonylurea; TZD, thiazolidinedione

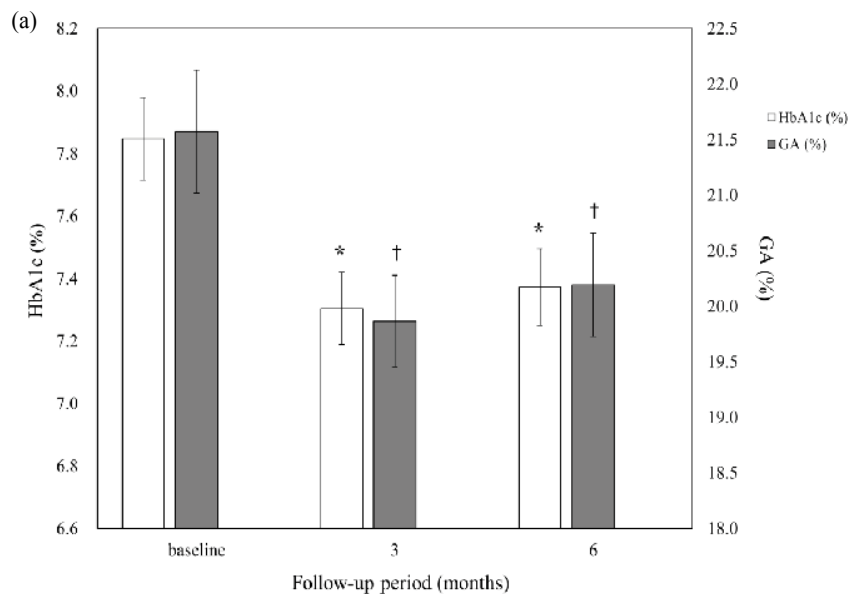

(b)

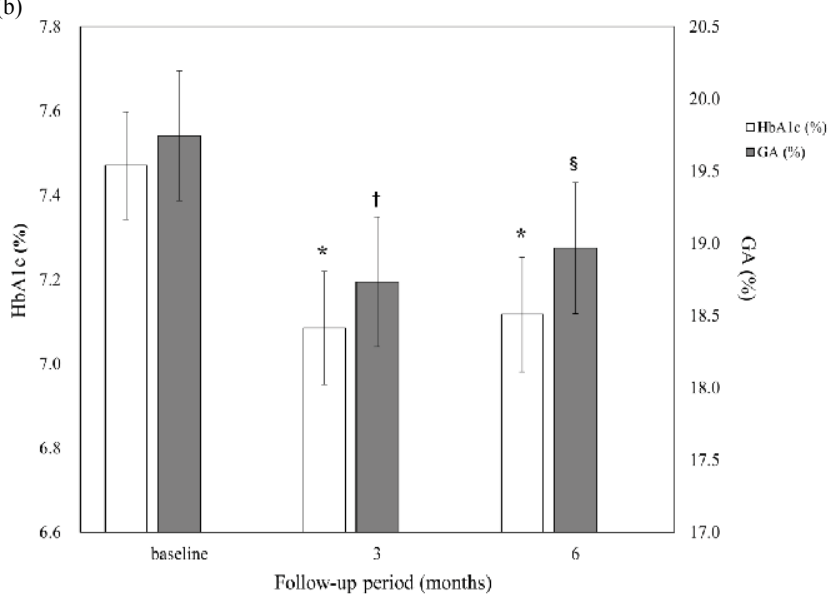

(c)

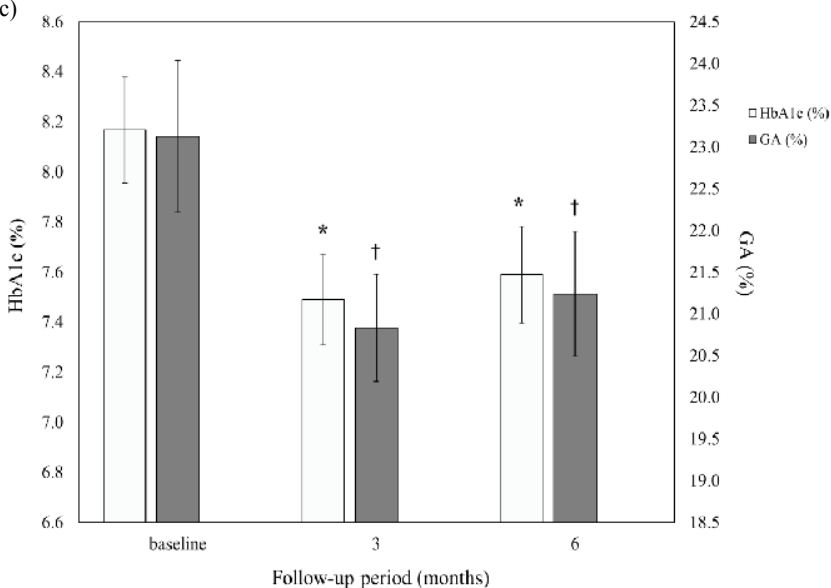

${ }^{*} P<0.01$ vs. baseline for $\mathrm{HbA} 1 \mathrm{c}$

${ }^{\dagger} P<0.01$ vs. baseline for GA

$\S P<0.05$ vs. baseline for $\mathrm{GA}$

a) Total patients $(n=127)$

b) Patients without diabetic nephropathy $(n=58)$

c) Patients with diabetic nephropathy $(n=69)$

$\mathrm{GA}$, glycoalbumin; $\mathrm{HbA} 1 \mathrm{c}$, hemoglobin A1c

Figure 1: Changes in hemoglobin $A 1 C$ and glycoalbumin levels after linagliptin administration.

Table 1: Baseline patient characteristics. 
Citation: Tanaka M, Nishimura T, Sekioka R, Kawai T, Meguro S, et al. (2016) Comparison of Glycemic Control Indicators and Safety Evaluation during Linagliptin Treatment over 6 Months in Japanese Type 2 Diabetic Patients with and without Nephropathy. J Diabetes Metab 7: 648. doi:10.4172/2155-6156.1000648

Page 4 of 6

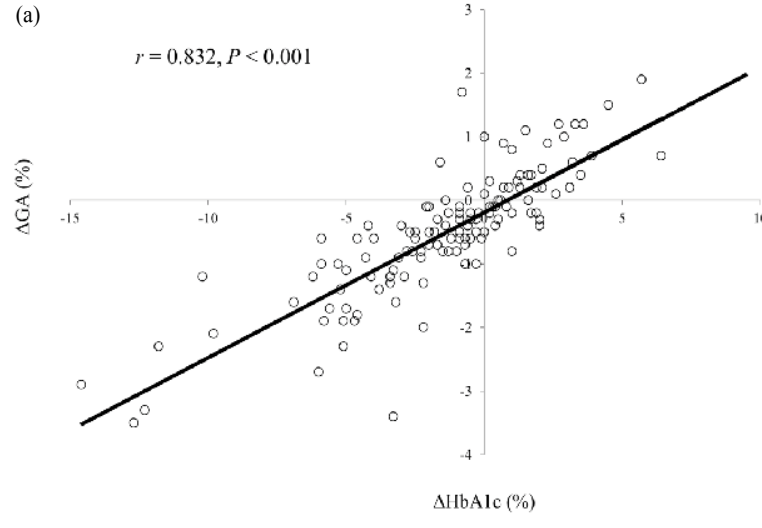

(b)

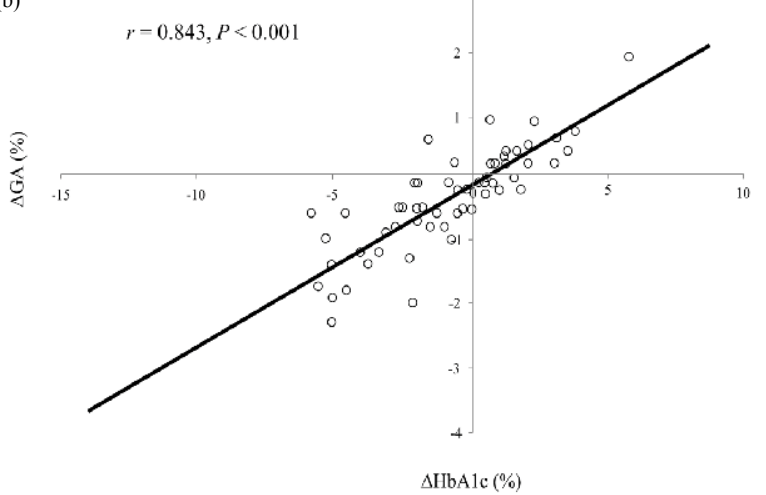

(c)

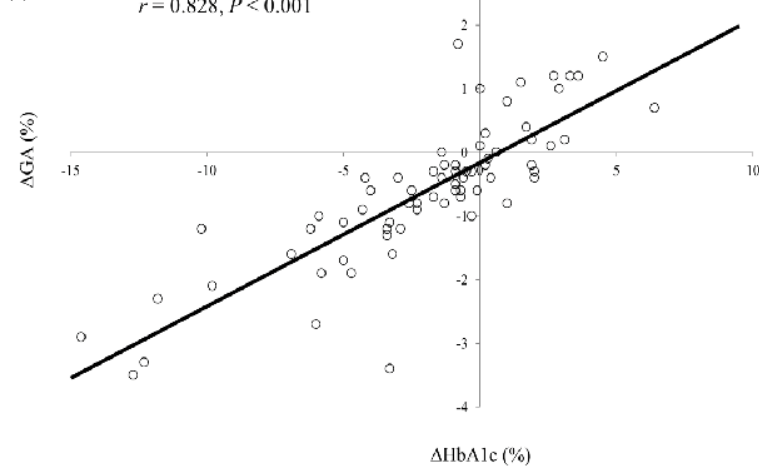

$\triangle \mathrm{HbA} 1 \mathrm{c}=\mathrm{HbA} 1 \mathrm{c}$ at 6 months - baseline $\mathrm{HbA} 1 \mathrm{c} ; \Delta \mathrm{GA}=\mathrm{GA}$ at 6 months - baseline GA

Horizontal axis shows $\triangle \mathrm{GA}$ and the vertical axis $\triangle \mathrm{HbA} 1 \mathrm{c}$

a) Total patients $(n=127)$

b) Patients without diabetic nephropathy $(n=58)$

c) Patients with diabetic nephropathy $(n=69)$

GA, glycoalbumin; HbA1c, hemoglobin A1c

Figure 2: Correlations between $\triangle \mathrm{HbA} 1 \mathrm{c}$ and $\triangle \mathrm{GA}$.

without nephropathy: $13.6 \pm 1.2 \mathrm{~g} / \mathrm{dL} \rightarrow 13.6 \pm 1.3 \mathrm{~g} / \mathrm{dL} \rightarrow 13.7 \pm 1.2 \mathrm{~g} /$ $\mathrm{dL}$; all $P=1.00$ vs. baseline).

Multivariate analysis of all patients showed that the factors contributing significantly to $\triangle \mathrm{HbAlc}$ were baseline $\mathrm{HbA1c}$ levels $(P=0.001)$ and diabetes duration $(P=0.007)$. Similarly, the factors contributing significantly to $\Delta \mathrm{GA}$ were baseline GA levels $(P<0.001)$ and diabetes duration $(P=0.017$, Table $2 \mathrm{a})$. In patients with diabetic nephropathy, baseline HbA1c levels $(P=0.002)$ and diabetes duration $(P=0.043)$ significantly contributed to $\triangle \mathrm{HbAlc}$, whereas only baseline GA levels $(P<0.001)$ contributed significantly to $\Delta \mathrm{GA}$ (Table $2 \mathrm{~b})$.

\section{Safety of linagliptin}

No significant changes in the eGFR after linagliptin administration were observed in the total patient group (Table 3a), in patients with diabetic nephropathy (Table $3 \mathrm{~b}$ ), or in those with reduced baseline eGFR (Figure 3). Moreover, clinical parameters did not change significantly after linagliptin administration in the total patient group (Table 3a). In patients with diabetic nephropathy, only triglyceride levels changed significantly after linagliptin administration (Table $3 \mathrm{~b}$ ).

(a) Total patients $(N=127)$

$\triangle \mathrm{HbA} 1 \mathrm{c}$

\begin{tabular}{|c|c|c|c|c|}
\hline & $\boldsymbol{\beta}$ & SE & $\boldsymbol{t}$ value & $\boldsymbol{p}$ value \\
\hline Sex (male = 1, female =0) & 0.045 & 0.202 & 0.22 & 0.826 \\
\hline Age & -0.004 & 0.009 & -0.400 & 0.69 \\
\hline Diabetes duration & 0.029 & 0.01 & 2.774 & $0.007^{*}$ \\
\hline BMI & 0.007 & 0.022 & 0.329 & 0.743 \\
\hline CPR index & 0.043 & 0.069 & 0.622 & 0.535 \\
\hline Baseline HbA1c & -0.265 & 0.079 & -3.359 & $0.001^{*}$ \\
\hline eGFR & 0.008 & 0.004 & 1.731 & 0.087 \\
\hline GGA & & & & \\
\hline Sex (male = 1, female $=0)$ & 0.056 & 0.681 & 0.082 & 0.935 \\
\hline Age & 0.046 & 0.031 & 1.484 & 0.142 \\
\hline Diabetes duration & 0.084 & 0.034 & 2.441 & $0.017^{*}$ \\
\hline BMI & -0.021 & 0.07 & -0.301 & 0.764 \\
\hline CPR index & -0.045 & 0.23 & -0.197 & 0.844 \\
\hline Baseline GA & -0.332 & 0.062 & -5.392 & $<0.001^{*}$ \\
\hline eGFR & 0.008 & 0.015 & 0.525 & 0.601 \\
\hline
\end{tabular}

(b) Patients with diabetic nephropathy $(n=69)$

$\triangle \mathrm{HbA} 1 \mathrm{c}$

\begin{tabular}{|c|c|c|c|c|}
\hline & $\boldsymbol{\beta}$ & SE & $\boldsymbol{t}$ value & $\boldsymbol{p}$ value \\
\hline Sex (male = 1, female =0) & 0.053 & 0.32 & 0.166 & 0.869 \\
\hline Age & -0.008 & 0.014 & -0.550 & 0.585 \\
\hline Diabetes duration & 0.03 & 0.015 & 2.082 & $0.043^{*}$ \\
\hline BMI & -0.007 & 0.034 & 0.194 & 0.847 \\
\hline CPR index & 0.215 & 0.186 & 1.152 & 0.256 \\
\hline Baseline HbA1c & -0.361 & 0.111 & -3.255 & $0.002^{*}$ \\
\hline eGFR & 0.003 & 0.008 & 0.384 & 0.703 \\
\hline
\end{tabular}

$\Delta \mathrm{GA}$

\begin{tabular}{|c|c|c|c|c|}
\hline & $\boldsymbol{\beta}$ & SE & $\boldsymbol{t}$ value & $\boldsymbol{p}$ value \\
\hline Sex (male = 1, female =0) & 0.928 & 1.064 & 0.106 & 0.388 \\
\hline Age & 0.09 & 0.049 & 0.261 & 0.071 \\
\hline Diabetes duration & 0.062 & 0.048 & 0.169 & 0.204 \\
\hline BMI & -0.080 & 0.107 & -0.101 & 0.460 \\
\hline CPR index & -0.288 & 0.646 & -0.080 & 0.658 \\
\hline Baseline GA & -0.353 & 0.088 & -0.632 & $<0.001^{*}$ \\
\hline eGFR & 0.009 & 0.027 & -0.053 & 0.737 \\
\hline
\end{tabular}

${ }^{*} P<0.05$

$\triangle \mathrm{HbA} 1 \mathrm{c}=\mathrm{HbA} 1 \mathrm{c}$ at 6 months - baseline $\mathrm{HbA} 1 \mathrm{c} ; \triangle \mathrm{GA}=\mathrm{GA}$ at 6 month - baseline $\mathrm{GA}$ $\mathrm{BMI}$, body mass index; CPR, C peptide immunoreactivity; CPR index $=100 \times$ serum CPR $(\mathrm{ng} / \mathrm{mL}) / \mathrm{blood}$ glucose $(\mathrm{mg} / \mathrm{dL}) ;$ eGFR, estimated glomerular filtration rate; GA, glycoalbumin; $\mathrm{HbA} 1 \mathrm{c}$, hemoglobin $\mathrm{A} 1 \mathrm{c}$; $\mathrm{SE}$, standard error

Table 2: Factors contributing to $\triangle \mathrm{HbA} 1 \mathrm{c}$ and $\triangle \mathrm{GA}$. 
Citation: Tanaka M, Nishimura T, Sekioka R, Kawai T, Meguro S, et al. (2016) Comparison of Glycemic Control Indicators and Safety Evaluation during Linagliptin Treatment over 6 Months in Japanese Type 2 Diabetic Patients with and without Nephropathy. J Diabetes Metab 7: 648. doi:10.4172/2155-6156.1000648

Page 5 of 6

(a) Total patients $(N=127)$

\begin{tabular}{|c|c|c|c|}
\hline & $\begin{array}{c}\text { Before } \\
\text { administration }\end{array}$ & $\begin{array}{c}\mathbf{6} \text { months after } \\
\text { administration }\end{array}$ & p value \\
\hline Body weight $(\mathrm{kg})$ & $66.1 \pm 13.2$ & $65.9 \pm 13.1$ & 0.675 \\
\hline AST $(\mathrm{U} / \mathrm{L})$ & $24.1 \pm 15.6$ & $25.7 \pm 24.0$ & 0.233 \\
\hline ALT (U/L) & $20.9 \pm 17.9$ & $21.0 \pm 18.7$ & 0.876 \\
\hline $\mathrm{Y}-\mathrm{GTP}(\mathrm{U} / \mathrm{L})$ & $52.4 \pm 113.4$ & $58.5 \pm 173.5$ & 0.549 \\
\hline eGFR $\left(\mathrm{mL} / \mathrm{min} / 1.73 \mathrm{~m}^{2}\right)$ & $54.2 \pm 23.2$ & $53.9 \pm 23.9$ & 0.570 \\
\hline $\mathrm{LDL}-\mathrm{C}(\mathrm{mg} / \mathrm{dL})$ & $104.5 \pm 31.6$ & $107.5 \pm 30.5$ & 0.134 \\
\hline $\mathrm{HDL}-\mathrm{C}(\mathrm{mg} / \mathrm{dL})$ & $48.3 \pm 12.9$ & $49.4 \pm 13.3$ & 0.064 \\
\hline $\mathrm{TG}(\mathrm{mg} / \mathrm{dL})$ & $169.7 \pm 138.3$ & $150.1 \pm 101.8$ & 0.098 \\
\hline $\mathrm{TC}(\mathrm{mg} / \mathrm{dL})$ & $188.5 \pm 45.3$ & $187.8 \pm 41.2$ & 0.803 \\
\hline $\mathrm{UA}(\mathrm{mg} / \mathrm{dL})$ & $5.80 \pm 1.53$ & $5.87 \pm 1.54$ & 0.369 \\
\hline $\mathrm{Hb}(\mathrm{g} / \mathrm{dL})$ & $13.18 \pm 1.81$ & $13.19 \pm 1.87$ & 0.958 \\
\hline
\end{tabular}

(b) Patients with diabetic nephropathy $(n=69)$

\begin{tabular}{|c|c|c|c|}
\hline & $\begin{array}{c}\text { Before } \\
\text { administration }\end{array}$ & $\begin{array}{c}\mathbf{6} \text { months after } \\
\text { administration }\end{array}$ & $\begin{array}{c}\boldsymbol{p} \\
\text { value }\end{array}$ \\
\hline Body weight $(\mathrm{kg})$ & $66.4 \pm 13.1$ & $66.2 \pm 13.0$ & 0.674 \\
\hline AST (U/L) & $23.8 \pm 17.6$ & $27.3 \pm 31.5$ & 0.123 \\
\hline $\mathrm{ALT}(\mathrm{U} / \mathrm{L})$ & $20.3 \pm 21.0$ & $20.7 \pm 22.3$ & 0.648 \\
\hline $\mathrm{V}-\mathrm{GTP}(\mathrm{U} / \mathrm{L})$ & $51.5 \pm 115.5$ & $72.9 \pm 243.9$ & 0.285 \\
\hline eGFR $\left(\mathrm{mL} / \mathrm{min} / 1.73 \mathrm{~m}^{2}\right)$ & $45.1 \pm 23.0$ & $44.0 \pm 24.1$ & 0.220 \\
\hline $\mathrm{LDL}-\mathrm{C}(\mathrm{mg} / \mathrm{dL})$ & $101.9 \pm 34.6$ & $105.7 \pm 29.5$ & 0.257 \\
\hline $\mathrm{HDL}-\mathrm{C}(\mathrm{mg} / \mathrm{dL})$ & $46.8 \pm 11.6$ & $48.4 \pm 11.9$ & 0.059 \\
\hline $\mathrm{TG}(\mathrm{mg} / \mathrm{dL})$ & $182.6 \pm 137.0$ & $153.5 \pm 108.6$ & 0.014 \\
\hline $\mathrm{TC}(\mathrm{mg} / \mathrm{dL})$ & $185.8 \pm 49.8$ & $184.3 \pm 42.5$ & 0.715 \\
\hline $\mathrm{UA}(\mathrm{mg} / \mathrm{dL})$ & $6.11 \pm 1.57$ & $6.14 \pm 1.54$ & 0.749 \\
\hline $\mathrm{Hb}(\mathrm{g} / \mathrm{dL})$ & $12.75 \pm 2.21$ & $12.70 \pm 2.28$ & 0.685 \\
\hline & & & \\
\hline
\end{tabular}

${ }^{*} P<0.05$

ALT, alanine aminotransferase; AST, aspartate aminotransferase; $\mathrm{Y}$-GTP gamma-glutamyl transpeptidase; eGFR, estimated glomerular filtration rate; $\mathrm{Hb}$ hemoglobin; LDL-C, low-density lipoprotein cholesterol; HDL-C, high-density lipoprotein cholesterol; TC, total cholesterol; TG, triglycerides; UA, uric acid

Table 3: Safety of linagliptin.

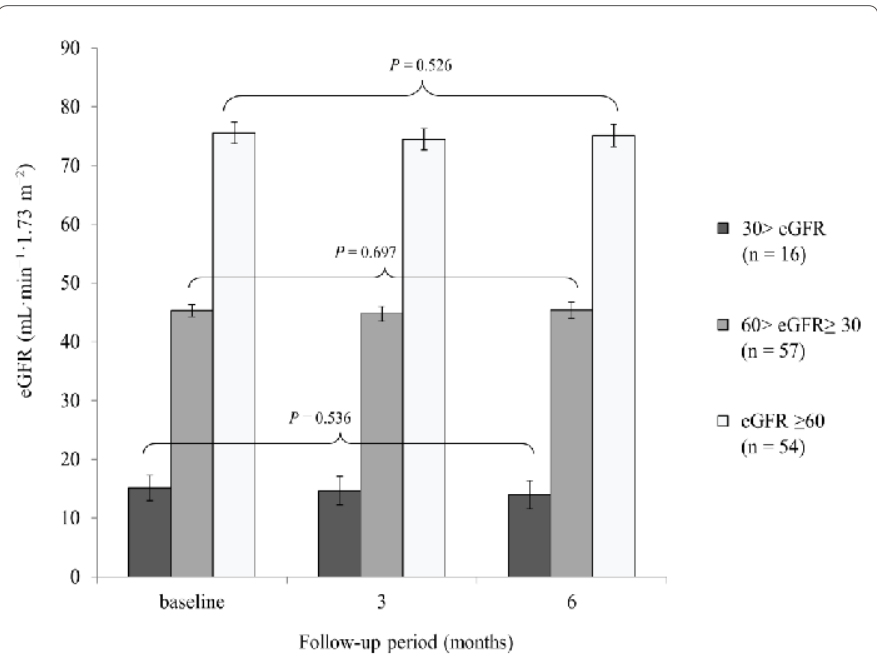

eGFR was categorized as $<30,30-60$, or $\geq 60 \mathrm{~mL} \cdot \mathrm{min}^{-1} \cdot 1.73 \mathrm{~m}^{-2}$ eGFR, estimated glomerular filtration rate

Figure 3: Changes in the estimated glomerular filtration rate.

\section{Discussion}

Linagliptin treatment significantly decreased $\mathrm{HbAlc}$ and GA levels at 3 months, and this hypoglycemic effect was sustained for 6 months. It is suggested that GA is equivalent to HbAlc as a glycemic control indicator for two reasons. First, the changes in GA levels after linagliptin administration were similar to those in HbAlc in our study. Second, we observed strong correlations between $\triangle \mathrm{GA}$ and $\triangle \mathrm{HbAlc}$ in all patient group (all patients, patients without nephropathy, and patients with nephropathy). When the patients were divided into 2 groups according to their hemoglobin levels (less than $13 \mathrm{~g} / \mathrm{dL}$ vs. 13 $\mathrm{g} / \mathrm{dL}$ or above), HbA1c and GA changed in a similar manner. Both glycemic control indicators changed more significantly in patients with lower hemoglobin levels.

GA is expected to be a more useful indicator of glycemic control than $\mathrm{HbAlc}$ in patients with severe renal impairment. In our cohort, the number of the patients with severe renal impairment was small ( 9 patients with stage 4 and 5 with stage 5 diabetic nephropathy, Table $1)$. This might be why changes in GA and HbAlc over time were similar in this study. If a similar investigation would be conducted in a population including many patients with severe renal dysfunction, the results might be different.

The results of our multivariate analyses showed that higher baseline HbAlc and GA levels were associated with improvements in the patients' blood glucose control, regardless of the complication of diabetic nephropathy. In 79 randomized controlled trials, including 20,503 patients who were administered DPP-4 inhibitors (including vildagliptin, sitagliptin, saxagliptin, linagliptin, and alogliptin), higher baseline $\mathrm{HbA1c}$ level was a significant predictor of the $\mathrm{HbA1c}$ lowering effect of DPP-4 inhibitor treatment [16]. These data are in agreement with our findings. On the other hand, baseline GA levels have been reported to be associated with the GA-lowering effect of a DPP-4 inhibitor only in one small study [17]. Our results suggest that both baseline GA and HbAlc levels can serve as predictors of blood glucose improvement after linagliptin administration, regardless of the presence of diabetic nephropathy.

The results of our multivariate analyses also showed that diabetes duration was significantly associated with both $\Delta \mathrm{GA}$ and $\Delta \mathrm{HbAlc}$. Thus, shorter diabetes duration is likely indicative of a favorable hypoglycemic effect of linagliptin administration. As longer diabetes duration is associated with reduced $\beta$-cell function, our findings suggest that linagliptin is more effective in patients who maintain $\beta$-cell function.

Linagliptin treatment improved $\mathrm{HbAlc}$ and GA levels regardless of the baseline eGFR and did not change the eGFR in the study population. In particular, linagliptin did not decrease the eGFR in patients with a low baseline eGFR. Linagliptin has been reported to be effective and safe in diabetes patients with mild $\left(\mathrm{eGFR}=60-90 \mathrm{~mL} \cdot \mathrm{min}^{-1} \cdot 1.73\right.$ $\left.\mathrm{m}^{-2}\right)$, moderate $\left(\mathrm{eGFR}=30-60 \mathrm{~mL} \cdot \mathrm{min}^{-1} \cdot 1.73 \mathrm{~m}^{-2}\right)$, and severe renal function impairment $\left(\mathrm{eGFR}<30 \mathrm{~mL} \cdot \mathrm{min}^{-1} \cdot 1.73 \mathrm{~m}^{-2}\right)$ [18,19]. A pharmacokinetics study of linagliptin showed large overlaps in the steady-state area under the curve and $\mathrm{C}_{\text {max }}$ values between subjects with normal renal function and those with renal function impairment [20]. Therefore, linagliptin is expected to be effective and safe in patients with a normal or low eGFR.

Our study has several limitations. First, patients not taking linagliptin were not included, i.e., we did not have a control group. Compensating for this shortcoming, we found an article reporting that linagliptin achieved consistent placebo-corrected $\mathrm{HbAlc}$ improvement across the three renal function categories; eGFR $>90 \mathrm{ml} / \mathrm{min} / 1.73 \mathrm{~m}^{2}$ : $-0.63 \%, 60-<90 \mathrm{ml} / \mathrm{min} / 1.73 \mathrm{~m}^{2}:-0.67 \%$, and $30-<60 \mathrm{ml} / \mathrm{min} / 1.73$ $\mathrm{m}^{2}:-0.53 \%$ [18]. Second, because of the retrospective design and 
Citation: Tanaka M, Nishimura T, Sekioka R, Kawai T, Meguro S, et al. (2016) Comparison of Glycemic Control Indicators and Safety Evaluation during Linagliptin Treatment over 6 Months in Japanese Type 2 Diabetic Patients with and without Nephropathy. J Diabetes Metab 7: 648. doi:10.4172/2155-6156.1000648

Page 6 of 6

limited number of patients, the existence of biases and confounding factors cannot be ruled out. Third, the results may not be applicable to the general population or patients with type 2 diabetes in primary care settings, because the patients who attended the university hospital might be a distinct patient group. Therefore, further prospective studies with larger sample sizes are required to confirm the present findings.

\section{Conclusions}

Linagliptin decreases HbAlc and GA levels regardless of the presence of diabetic nephropathy. HbA1c and GA levels are equivalent indicators of glycemic control during linagliptin treatment. High baseline HbAlc and GA levels might be good predictors of patients' responsiveness to linagliptin treatment regardless of the presence of diabetic nephropathy.

\section{References}

1. Derosa G, Maffioli $P$ (2012) Dipeptidyl peptidase-4 inhibitors: 3 years of experience. Diabetes Technol Ther 14: 350-364.

2. Hirakawa $\mathrm{Y}$, Arima $\mathrm{H}$, Zoungas $\mathrm{S}$, Ninomiya T, Cooper M, et al. (2014) Impact of visit-to-visit glycemic variability on the risks of macrovascular and microvascular events and all-cause mortality in type 2 diabetes: the ADVANCE trial. Diabetes Care 37: 2359-2365.

3. Erlinger TP, Brancati FL (2001) Postchallenge hyperglycemia in a national sample of U.S. adults with type 2 diabetes. Diabetes Care 24: 1734-1738.

4. Bonora E, Corrao G, Bagnardi V, Ceriello A, Comaschi M, et al. (2006) Prevalence and correlates of post-prandial hyperglycaemia in a large sample of patients with type 2 diabetes mellitus. Diabetologia 49: 846-854.

5. Yoshiuchi K, Matsuhisa M, Katakami N, Nakatani Y, Sakamoto K, et al. (2008) Glycated albumin is a better indicator for glucose excursion than glycated hemoglobin in type 1 and type 2 diabetes. Endocr J 55: 503-507.

6. Suwa T, Ohta A, Matsui T, Koganei R, Kato H, et al. (2010) Relationship between clinical markers of glycemia and glucose excursion evaluated by continuous glucose monitoring (CGM). Endocr J 57: 135-140.

7. Freedman BI, Shihabi ZK, Andries L, Cardona CY, Peacock TP, et al. (2010) Relationship between assays of glycemia in diabetic subjects with advanced chronic kidney disease. Am J Nephrol 31: 375-379.

8. Viljoen M, de Oliveira AA, Milne FJ (1991) Physical properties of the red blood cells in chronic renal failure. Nephron 59: 271-278.
9. Scheen AJ (2010) Pharmacokinetics of dipeptidylpeptidase-4 inhibitors. Diabetes Obes Metab 12: 648-658.

10. Graefe-Mody U, Friedrich C, Port A, Ring A, Retlich S, et al. (2011) Effect of renal impairment on the pharmacokinetics of the dipeptidyl peptidase-4 inhibitor linagliptin(*). Diabetes Obes Metab 13: 939-946.

11. Tanaka M, Sekioka R, Nishimura T, Kawai T, Meguro S, et al. (2015) Serum C-peptide to plasma glucose ratio may be associated with efficacy of vildagliptin in Japanese patients with type 2 diabetes mellitus. Diabetol Int 6: 197-205.

12. Davis MD, Kern TS, Rand LI (1997) Diabetic retinopathy. International textbook of diabetes mellitus. ( $\left.2^{\text {nd }} e d n\right)$, John Wiley \& Sons.

13. Haneda M, Utsunomiya K, Koya D, Babazono T, Moriya T, et al. (2015) A new classification of Diabetic Nephropathy 2014: A report from Joint Committee on Diabetic Nephropathy. Clin Exp Nephrol 19: 1-5.

14. Committee of the Japan Diabetes Society on the Diagnostic Criteria of Diabetes Mellitus, Seino Y, Nanjo K, Tajima N, Kadowaki T, et al. (2010) Report of the committee on the classification and diagnostic criteria of diabetes mellitus. J Diabetes Investig 1: 212-228.

15. Matsuo S, Imai E, Horio M, Yasuda Y, Tomita K, et al. (2009) Revised equations for estimated GFR from serum creatinine in Japan. Am J Kidney Dis 53: $982-$ 992.

16. Esposito K, Chiodini P, Capuano A, Maiorino MI, Bellastella G, et al. (2014) Baseline glycemic parameters predict the hemoglobin A1c response to DPP4 inhibitors: Meta-regression analysis of 78 randomized controlled trials with 20,053 patients. Endocrine 46: 43-51.

17. Ito H, Mifune M, Matsuyama E, Furusho M, Omoto T, et al. (2013) Vildagliptin is Effective for Glycemic Control in Diabetic Patients Undergoing either Hemodialysis or Peritoneal Dialysis. Diabetes Ther 4: 321-329.

18. Groop PH, Del Prato S, Taskinen MR, Owens DR, Gong Y, et al. (2014) Linagliptin treatment in subjects with type 2 diabetes with and without mild-tomoderate renal impairment. Diabetes Obes Metab 16: 560-568.

19. McGill JB, Sloan L, Newman J, Patel S, Sauce C, et al. (2013) Long-term efficacy and safety of linagliptin in patients with type 2 diabetes and severe renal impairment: a 1-year, randomized, double-blind, placebo-controlled study. Diabetes Care 36: 237-244.

20. Giorda CB, Nada E, Tartaglino B (2014) Pharmacokinetics, safety, and efficacy of DPP-4 inhibitors and GLP-1 receptor agonists in patients with type 2 diabetes mellitus and renal or hepatic impairment. A systematic review of the literature. Endocrine 46: 406-419. 